American Journal of Applied Sciences 5 (6): 659-664, 2008

ISSN 1546-9239

(C) 2008 Science Publications

\title{
Performance Comparison of Two On-demand Routing Protocols for Ad-hoc Networks based on Random Way Point Mobility Model
}

\author{
Geetha Jayakumar and G. Gopinath \\ Department of Computer Science, Bharathidasan University, \\ Tiruchirapalli-620 023, India
}

\begin{abstract}
Ad hoc networks are characterized by multihop wireless connectivity, frequently changing network topology and the need for efficient dynamic routing protocols plays an important role. We compare the performance of two prominent on-demand routing protocols for mobile ad hoc networks: Dynamic Source Routing (DSR), Ad Hoc On-demand distance Vector Routing (AODV). A detailed simulation model with MAC and physical layer models is used to study the interlayer interactions and their performance implications. We demonstrate that even though DSR and AODV share similar ondemand behavior, the differences in the protocol mechanisms can lead to significant performance differentials. In this paper we examine two on demand routing protocols AODV and DSR based on packet delivery ratio, normalized routing load, normalized MAC load, average end to end delay by varying the number of sources, speed and pause time.
\end{abstract}

Keywords: MANET, routing protocol, on-demand, DSR, AODV, random way point model

\section{INTRODUCTION}

A network is a collection of two or more computing devices, which is connected by a communication medium. There are two types of network wired network and wireless network. Figure 1 below shows a simple wired network with three computing devices. When a node wishes to send information to the destination, it may do so by transmitting the information along a shared communication medium. In this paper any device actively participating in the network is called a node. Nodes are connected by communication medium or link. The nodes exchange information over links in discrete blocks called packets. If nodes do not have a direct link, then the packets have to traverse the path through the intermediate nodes. Whenever packet is transmitted from one node to another, it is said to have a hop. The various nodes within the network must cooperate with each other to make the information exchange successful. This cooperation process is called routing.

Wireless networking is an emerging technology that allows users to access information and services electronically, regardless of their geographic position.

Wireless networks can be classified in two types:

1. Infrastructured networks.

2. Infrastructureless (Ad hoc) networks ${ }^{[1]}$

An ad hoc networks or infrastructureless networks is a collection of mobile nodes which forms a temporary network without the aid of centralized administration or standard support devices regularly available in conventional networks. In this paper, it is assumed that the mobile hosts uses wireless RF transceivers as their network interface. Routing protocol plays an important role if two hosts wishes to exchange packets which may not be able to communicate directly. All nodes are mobile and can be connected dynamically in an arbitrary manner. All nodes of these networks behave as routers and take part in discovery and maintenance of routes to other nodes in the network. This situation becomes more complicated if more nodes are added within the network. An Ad-Hoc routing protocol must be able to decide the best path between the nodes, minimize the bandwidth overhead to enable proper routing, minimize the time required to converge after the topology changes. Ad hoc networks are very useful in emergency search-and-rescue operations, meetings or conventions in which persons wish to quickly share information, and data acquisition operations in inhospitable terrain.

This ad-hoc routing protocols can be divided into two categories:

Table-driven routing protocols: In table driven routing protocols, consistent and up-to-date routing information to all nodes is maintained at each node.

On-Demand routing protocols: In On-Demand routing protocols, the routes are created as and when

Corresponding Author: Geetha Jayakumar, Department of Computer Sciences, Bharathidasan University, Tiruchirapalli-620023, India Tel: +91-431-2331663 Fax: +91-431-2331662 
required. When a source wants to send to a destination, it invokes the route discovery mechanisms to find the path to the destination. Once a Route has been established, it is maintained until either the destination becomes inaccessible (along every path from the source), or until the route is no longer used, or expired $^{[2]}$.

In recent years, a variety of new routing protocols targeted specifically at this environment have been developed. We consider two wirelesses on demand ad hoc network routing protocols that cover a range of design choices.

Dynamic Source Routing (DSR): The key distinguishing feature of DSR ${ }^{[3,4]}$ is the use of source routing. Dynamic Source Routing (DSR) ${ }^{[5]}$ is a reactive protocol i.e. it doesn't use periodic advertisements. It computes the routes when necessary and then maintains them. Source routing is a routing technique in which the sender of a packet determines the complete sequence of nodes through which the packet has to pass, the sender explicitly lists this route in the packet's header, identifying each forwarding hop by the address of the next node to which to transmit the packet on its way to the destination host.

Ad Hoc On-Demand Distance Vector Routing (AODV): Ad hoc On-demand Distance Vector (AODV) ${ }^{[6]}$ is essentially a combination of both DSR and DSDV. It borrows the basic on-demand mechanism of Route Discovery and Route Maintenance from DSR, plus the use of hop-by-hop routing, sequence numbers, and periodic beacons from DSDV. It uses destination sequence numbers to ensure loop freedom at all times and by avoiding the Bellman-Ford count-to-infinity problem offers quick convergence when the ad hoc network topology changes

In this research paper we attempted to present an overview of two main categories of mobile ad-hoc routing protocols and performance comparison of both the protocols based on Random way point model and the simulation of two routing protocols focussing on their differences in their dynamic behaviours that can lead to performance differences.

\section{SIMULATION MODEL}

A detailed simulation model based on $n s-2^{[7]}$ is used in the evaluation. The Distributed Coordination Function (DCF) of IEEE $802.11^{[8]}$ for wireless LANs is used as the MAC layer protocol. An unslotted carrier sense multiple access (CSMA) technique with collision avoidance (CSMA/CA) is used to transmit the data packets. The radio model uses characteristics similar to a commercial radio interface, Lucent's WaveLAN. WaveLAN ${ }^{[9,10]}$ is modeled as a shared-media radio with a nominal bit rate of $2 \mathrm{Mb} / \mathrm{s}$ and a nominal radio range of $250 \mathrm{~m}^{[11,12]}$.

The protocols maintain a send buffer of 64 packets. It contains all data packets waiting for a route, such as packets for which route discovery has started, but no reply has arrived yet. To prevent buffering of packets indefinitely, packets are dropped if they wait in the send buffer for more than $30 \mathrm{~s}$. All packets (both data and routing) sent by the routing layer are queued at the interface queue until the MAC layer can transmit them. The interface queue has a maximum size of 50 packets and is maintained as a priority queue with two priorities each served in FIFO order. Routing packets get higher priority than data packets.

Traffic and Mobility models: In this paper we are using traffic and mobility model based on Continuous bit rate (CBR) traffic sources are used. The sourcedestination pairs are spread randomly over the network. Only 512-byte data packets are used. The number of source-destination pairs and the packet sending rate in each pair is varied to change the offered load in the network.

The mobility model uses the random waypoint model ${ }^{[13]}$ in a rectangular field. The field configurations used is: $500 \mathrm{~m} \times 500 \mathrm{~m}$ field with 50 nodes. Here, each packet starts its journey from a random location to a random destination with a randomly chosen speed (uniformly distributed between $0-20 \mathrm{~m} / \mathrm{s}$ ). Once the destination is reached, another random destination is targeted after a pause. The pause time, which affects the relative speeds of the mobiles, is also varied. Simulations are run for 100 simulated seconds. Identical mobility and traffic scenarios are used across protocols to gather fair results.

\section{PERFORMANCE METRICS}

The following four important performance metrics are considered for evaluation of these two on demand routing protocols.

Packet delivery fraction: The ratio of the data packets delivered to the destinations to those generated by the CBR sources. 
Average end-to-end delay of data packets: This includes all possible delays caused by buffering during route discovery latency, queuing at the interface queue, retransmission delays at the MAC, and propagation and transfer times.

Normalized routing load: The number of routing packets transmitted per data packet delivered at the destination. Each hop-wise transmission of a routing packet is counted as one transmission

Normalized MAC load: The number of routing, Address resolution protocol (ARP), and control (e.g., RTS, CTS, ACK) packets transmitted by the MAC layer for each delivered data packet. Essentially, it considers both routing overhead and the MAC control overhead. Like normalized routing load, this metric also accounts for transmission at every hop.

The first two metrics are the most important for best effort traffic. The routing load metric evaluates the efficiency of the routing protocol. Finally the MAC load is a measure of effective utilization of the wireless medium by data traffic.

\section{RESULTS AND DISCUSSIONS}

The simulation parameters which have been considered for doing the performance comparison of two ondemand routing protocols is given below.

\begin{tabular}{ll}
\hline Protocols & AODV, DSR \\
\hline Simulation time & 100 seconds \\
\#of nodes & 50 \\
Map size & $500 \mathrm{mx} 500 \mathrm{~m}$ \\
Max speed & $20 \mathrm{~m} / \mathrm{s}$ \\
Mobility model & Random way point \\
Traffic Type & Constant bit rate $(\mathrm{CBR})$ \\
Packet Size & 512 bytes \\
Connection rate & 4 pkts $/ \mathrm{sec}$ \\
Pause time & $0,10,20,40,100$ \\
\#of connections & $10,20,30,40$ \\
\hline
\end{tabular}

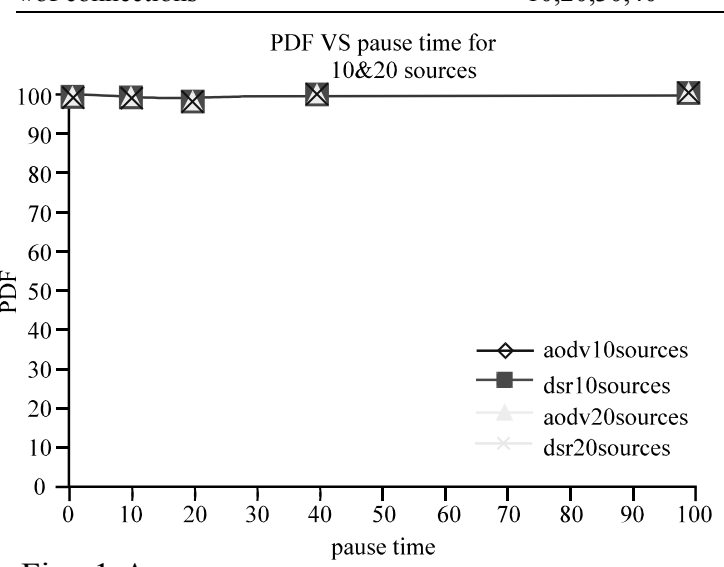

Fig. 1-A

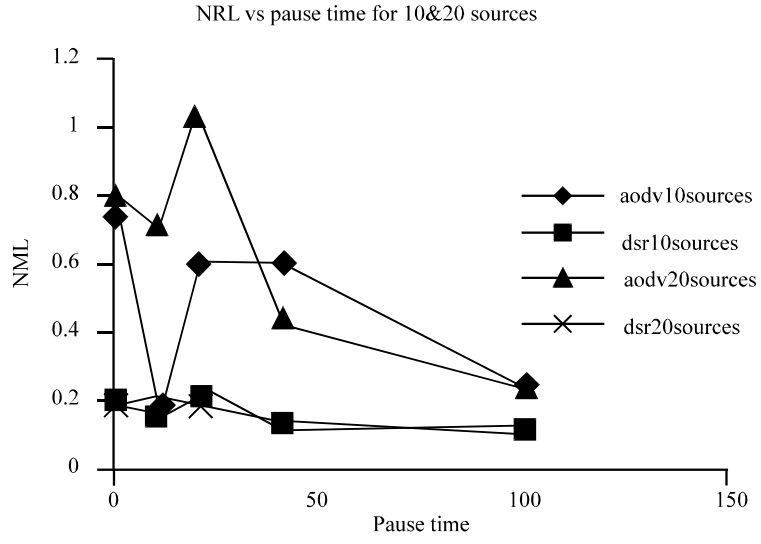

Fig. 1-B

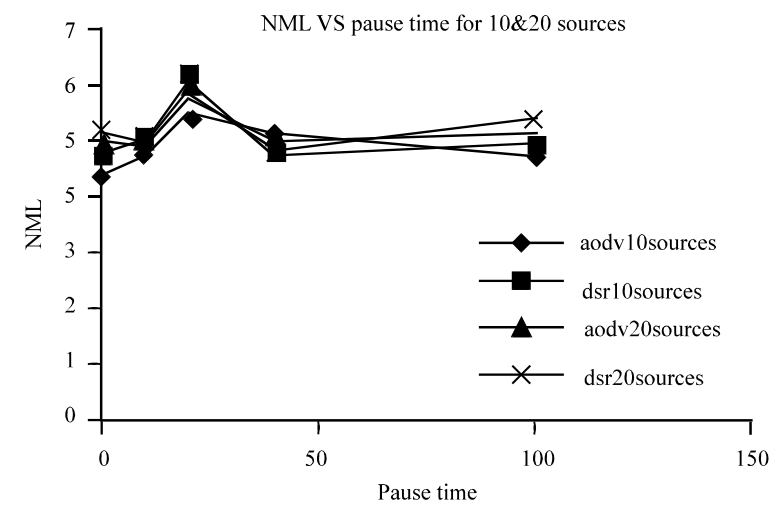

Fig. 1-C

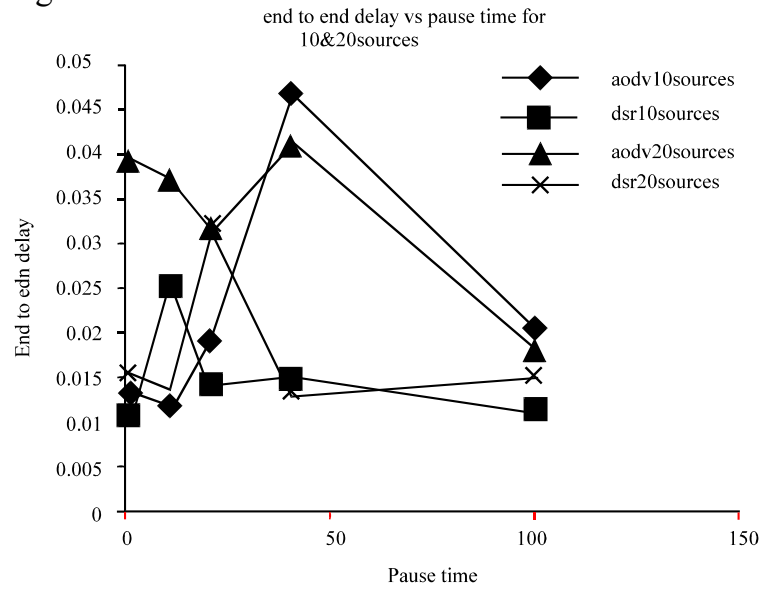

Fig. 1. D

This simulation analysis is made from the Fig. 1 for 10 sources. First we analyze the first parameter Packet delivery ratio with respect varied pause times. Figs shows that the packet delivery ratio for the two ondemand routing protocol is similar. Normalized 
Am. J. Applied Sci., 5 (6): 659-664, 2008

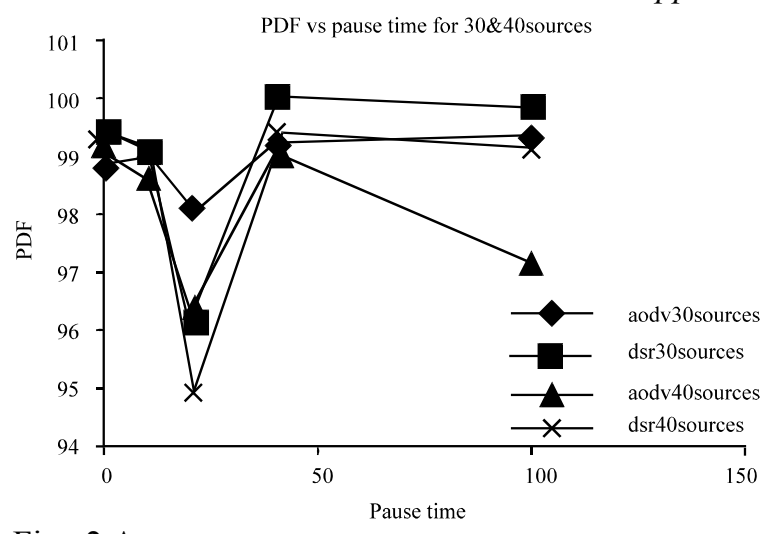

Fig. 2.A

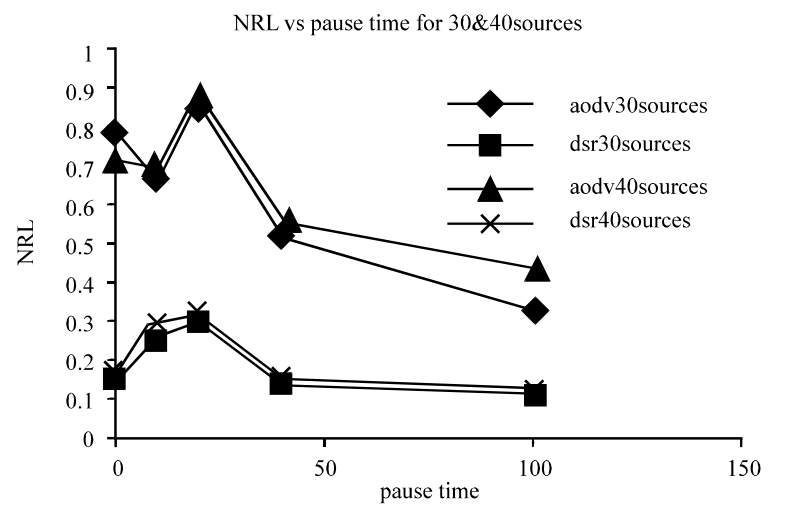

Fig. 2.B

routing load with varied pause times is analyzed and it is found that for DSR it is less when compared to AODV and we see that it is fairly stable even with increase number of sources. A relatively stable normalizes routing load is a desirable property for scalability of the protocols. We find that major contribution to AODV routing overhead is from

route requests, while route replies constitute a large fraction of DSR routing overhead. By virtue of aggressive caching, DSR is more likely to find the route in the cache and hence the route discovery process

occurs less frequently than AODV and hence the routing overhead for DSR is less when compared to AODV. The third parameter Normalized MAC load is analyzed with respect to different pause times and it is found that for AODV it is less when compared to DSR for lower pause times. This is because RERRs are handled different in each protocol. RERR are unicast in DSR, and therefore contribute to additional MAC overhead like RREPs. In AODV, RERRs are broadcast like RREQs and hence are less expensive. Consequently when the MAC overhead is factored DSR is found to generate higher overall network load than AODV in all scenarios despite having less routing

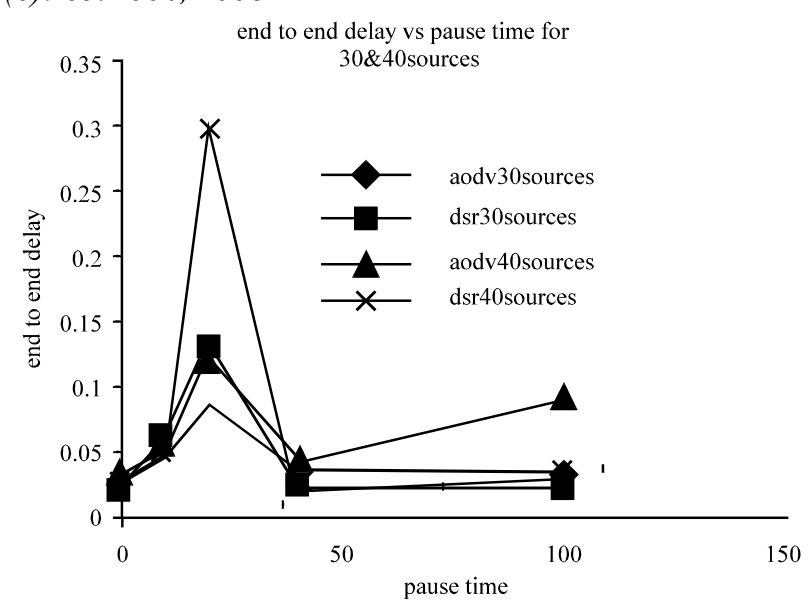

Fig. 2.C

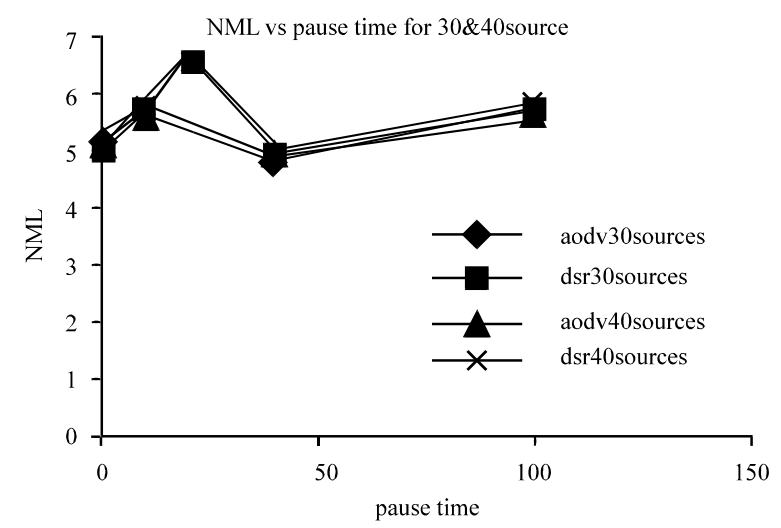

Fig. 2.D

overhead. With respect to fourth parameter when analyzed the delay AODV and DSR have identical delays for 10 sources.

The simulation analysis for the Fig. 1 for 20 sources shows that the packet delivery ratio with respect to varied pause times for both the protocols looks similar. The Normalized routing load for DSR is found to be less when compared to AODV because of DSR aggressive caching technique. The Normalized MAC load for AODV is slightly lesser when compared to DSR. The end to end delays for both the protocols looks identical.

The simulation analysis for the Fig. 2 for 30 sources shows that the packet delivery ratio with respect to varied pause times for both the protocols looks similar. The Normalized routing load with respect to varied pause times for DSR is found to be very less when compared to AODV because of DSR aggressive caching technique. The Normalized MAC load for AODV is slightly lesser when compared to DSR. With respect to end to end delays in the case of 30 sources AODV has less delay than DSR for lower pause times. 
But for higher pause times DSR has less delay when compared to AODV.

The simulation analysis for the Fig. 3 for 40 sources shows that the packet delivery ratio with respect to varied pause times for both the protocols looks similar. The Normalized routing load with respect to varied pause times for DSR is found to be very less when compared to AODV because of DSR aggressive caching technique. The Normalized MAC load for AODV is slightly lesser when compared to DSR. With respect to end to end delays in the case of 40 sources AODV has less delay than DSR for lower pause times. But for higher pause times DSR has less delay when compared to AODV.

\section{OBSERVATIONS}

The simulation results bring out some important characteristic of differences between the two on demand routing protocols. The presence of high mobility implies frequent link failures and each routing protocol reacts differently during link failures. The different basic working mechanism of these protocols leads to the differences in their performances.

For DSR and AODV, packet delivery ratio is independent of offered traffic load, with both protocols delivering between $85 \%$ and $100 \%$ of the packets in all cases. In contrast, the lazy approach used by the ondemand protocols, AODV and DSR to build the routing information as and when they are created make them more adaptive and result in better performance (high packet delivery fraction and lower average end-to-end packet delays).

Next the simulation results compare the performances of AODV and DSR lead us to the following conclusions.

Effect of Mobility: In the presence of high mobility, link failures can happen very frequently. Link failures trigger new route discoveries in AODV since it has at most one route per destination in its routing table. Thus, the frequency of route discoveries in AODV is directly proportional to the number of route breaks. The reaction of DSR to link failures in comparison is mild and causes route discovery less often. The reason is the abundance of cached routes at each node. Thus, the route discovery is delayed in DSR until all cached routes fail. But with high mobility, the chance of the caches being stale is quite high in DSR. Eventually when a route discovery is initiated, the large number of replies received in response is associated with high MAC overhead and cause increased interference to data traffic. Hence, the cache staleness and high MAC overhead together result in significant degradation in performance for DSR in high mobility scenarios.

In lower mobility scenarios, DSR often performs better than AODV, because the chances of find the route in one of the caches is much higher. However, due to the constrained simulation environment (lesser simulation time and lesser mobility models), the better performance of DSR over AODV couldn't be observed.

Routing load effect: DSR almost always has a lower routing load than AODV. This can be attributed to the caching strategy used by DSR. By virtue of aggressive caching, DSR is more likely to find a route in the cache, and hence resorts to route discovery less frequently than AODV.

\section{CONCLUSIONS}

In this paper we have compared the performance of AODV and DSR routing protocols for ad hoc networks using ns-2 simulations. Unfortunately, TORA simulations couldn't be successfully carried out.

AODV and DSR use the reactive On-demand routing strategy. Both AODV and DSR perform better under high mobility simulations. High mobility results in frequent link failures and the overhead involved in updating all the nodes with the new routing information as in DSDV is much more than that involved AODV and DSR, where the routes are created as and when required.

DSR and AODV both use on-demand route discovery, but with different routing mechanics. In particular, DSR uses source routing and route caches, and does not depend on any periodic or timer-based activities. DSR exploits caching aggressively and maintains multiple routes per destination. AODV, on the other hand, uses routing tables, one route per destination, and destination sequence numbers, a mechanism to prevent loops and to determine freshness of routes. The general observation from the simulation is that for application-oriented metrics such as packet delivery fraction and delay. AODV, outperforms DSR in more "stressful" situations (i.e., smaller number of nodes and lower load and/or mobility), with widening performance gaps with increasing stress (e.g., more load, higher mobility). DSR, however, consistently generates less routing load than AODV. The poor performances of DSR are mainly attributed to aggressive use of caching, and lack of any mechanism to expire stale routes or determine the freshness of routes when multiple choices are available. Aggressive caching, however, seems to help DSR at low loads and 
also keeps its routing load down. If there could be any mechanisms to expire routes and or determine the freshness of routes in the route cache could benefit DSR performance significantly. It is found that for lower loads DSR is more effective while AODV is more effective for higher loads.

\section{REFERENCES}

1. Stallings. W., 1996, Local \& Metropolitan Area Networks, Prentice Hall, Englewood Cliffs, NJ

2. Elizabeth, Belding-Royer, 2003, Routing approaches in mobile Ad Hoc networks, in: S. Basagni, M. Conti, S. Giordano, I. Stojemenvoic (Eds), Ad Hoc Networking, IEEE Press Wiley, New York.

3. Broch J., D. Johnson and D. Maltz, 1999, The Dynamic Source Routing Protocol for Mobile Ad Hoc Networks, http://www.ietf.org/internetdrafts/draft-ietf-manet-dsr-03.txt, IETF Internet draft, Oct., 1999.

4. Johnson., D. and D. Maltz, 1996. Dynamic Source Routing in Ad Hoc Wireless Networks, T. Imielinski and H. Korth, (Eds.) Mobile Computing, Ch.5.Kluwer.

5. David B. Johnson, A. David Maltz, and Yih-Chun $\mathrm{Hu}$, The Dynamic Source Routing Protocol for Mobile Ad Hoc Networks (DSR), draft-ietf-manet-dsr-10.txt> Internet-draft, 19 July 2004.
6. Perkins, C., E. Belding-Royer and S. Das, 2003. Ad hoc On-Demand Distance Vector (AODV) Routing, RFC 3561

7. Fall, K. and K. Vardhan, (Eds.) ns notes and documentation, 1999; available from http://wwwmash.cd.berkeley.edu/ns/.

8. IEEE, 1997, Wireless LAN Medium Access Control (MAC) and Physical layer (PHY) Specifications, IEEE Std. 802.11-1997

9. Eckhardt, D. and P. Steenkiste, 1996. Measurement and Analysis of the Error Characteristics of an In-building Wireless Network, Proc. ACM SIG-COMM '96, pp: 243-54.

10. Tuch, B., 1993, Development of WaveLAN, an ISM Band Wireless LAN, AT\&T Tech. J.,vol. 72, no. 4, pp: 27-33.

11. Anastasi, G., E. Borgia, M. Conti, E. Gregori, 2003. IEEE 802.11 Ad Hoc networks: performance measurements, Proceedings of the Workshop on Mobile and Wireless Networks (MWN 2003).

12. Anastasi, G., E. Borgia, M. Conti, E. Gregori, 2003. IEEE 802.11 Ad Hoc networks: protocols, performance and open issues, in: S.Basagni, M.Conti, S.Giordano, I.Stojmenvoic (Eds.), Ad hoc Networking, IEEE Press Wiley, New York

13. Broch J., 1998, A Performance Comparison of Multihop Wireless Ad Hoc Network Routing Protocols, Proc. IEEE/ACM MOBICOM 98, pp: 85-97. 